\title{
Narrativa
}

\section{Il vuoto del maschio. Stereotipi e anti-stereotipi della maschilità nei romanzi di Natalia Ginzburg}

\section{Beatrice Manetti}

\section{(2) OpenEdition}

\section{Journals}

\section{Edizione digitale}

URL: https://journals.openedition.org/narrativa/544

DOI: $10.4000 /$ narrativa. 544

ISSN: 2804-1224

\section{Editore}

Presses universitaires de Paris Nanterre

\section{Edizione cartacea}

Data di pubblicazione: 1 décembre 2018

Paginazione: 117-127

ISBN: 978-2-84016-325-1

ISSN: 1166-3243

Notizia bibliografica digitale

Beatrice Manetti, «ll vuoto del maschio. Stereotipi e anti-stereotipi della maschilità nei romanzi di Natalia Ginzburg», Narrativa [Online], 40 | 2018, online dal 01 novembre 2021, consultato il 08 décembre 2021. URL: http://journals.openedition.org/narrativa/544 ; DOI: https://doi.org/10.4000/ narrativa. 544

Narrativa est mise à disposition selon les termes de la Licence Creative Commons Attribution 4.0 International. 


\title{
Il vuoto del maschio. Stereotipi e anti-stereotipi della maschilità nei romanzi di Natalia Ginzburg
}

\begin{abstract}
RiASSUNTO
Nella riflessione sulle identità e sui ruoli di genere che attraversa l'intera produzione narrativa di Natalia Ginzburg, la figura dell'omosessuale svolge un ruolo fondamentale sia sul piano "ideologico", nella ridefinizione dei modelli di maschilità, sia dal punto di vista narratologico, nella costruzione e nel funzionamento del sistema dei personaggi. Il saggio ne indaga la caratterizzazione e ne segue gli sviluppi nell'arco di trent'anni, nei tre romanzi in cui occupa una posizione centrale: Valentino (1957), Caro Michele (1973) e La città e la casa (1984).

\section{RÉSUMÉ}

Dans la réflexion sur les identités et les rôles de genre qui traverse toute la production narrative de Natalia Ginzburg, la figure de l'homosexuel joue un rôle fondamental tant au niveau "idéologique", dans la redéfinition des modèles de masculinité, que d'un point de vue narratologique, dans la construction et le fonctionnement du système des personnages. Cet article examine sa caractérisation et suit ses développements sur une période de trente ans, dans les trois romans dans lesquels il occupe une place centrale : Valentino (1957), Caro Michele (1973) et La città e la casa (1984).
\end{abstract}



ulla Stampa del 10 dicembre 1977, con una scelta sorprendente in un'autrice che ha sempre esibito una tenace sordità, quando non un aperto fastidio, nei confronti delle punte più politicamente estreme del movimento femminista ma che evidentemente non era insensibile alle sue elaborazioni teoriche, Natalia Ginzburg recensisce positivamente Nato di donna di Adrienne Rich, uscito quello stesso anno per Garzanti. La sorpresa si attenua, fino a scomparire, via via che si procede nella lettura. "Donne e uomini" obbedisce all'impianto argomentativo della recensione soltanto nelle prime 
righe, per trasformarsi rapidamente in un'interrogazione continuata, quasi un bilancio o una forma contratta di autocoscienza in pubblico.

Nel disvelamento e nell'analisi del proprio atteggiamento ambivalente nei confronti delle questioni di genere, Ginzburg tocca nodi generazionali, culturali e psicologici che non riguardano esclusivamente lei: l'interiorizzazione dei modelli patriarcali di maschilità come processo che struttura l'identità sessuale non solo degli uomini ma anche delle donne: "L'immagine virile che io ho in testa, è quella d'un uomo seduto in una poltrona, a leggere il giornale; stanco magari, per aver lavorato nel giorno, però comodamente seduto, mentre le donne lavano i piatti e badano ai bambini"; la difficoltà e insieme la necessità di rinunciarvi: "Erano attributi, se si vuole, del tutto imbecilli; però il fatto è che al posto di quelle immagini c'è oggi il vuoto"; il difficile compito di riempire quel vuoto con un'immagine nuova:

A chi spetta il compito di disegnare un'idea dell'uomo che abbia i connotati dei migliori uomini che abbiamo conosciuto, e che sia come essi erano o sono, però nuova? Multiforme, coraggiosa, estrosa, forte della forza dello spirito, e fragile della ammirevole fragilità e duttilità dell'ingegno? E strana, e tale da dare alle donne protezione paterna, un'incondizionata e pietosa comprensione, ma anche ambiguità, la chiarezza cristallina della volontà e insieme anche il mistero e le tenebre d'un pianeta diverso?

Con uno scarto ai limiti del paradosso, il discorso, che ha preso le mosse da un libro intitolato Nato di donna, approda a quella che per Ginzburg è la questione centrale dell'identità maschile: la reinvenzione e la riappropriazione della paternità. Da questo nucleo concettuale si diramano a cascata le domande che avevano impegnato l'autrice fino a quel momento, e che la terranno impegnata ancora per molti anni, intorno alla fluidità multiforme delle identità e delle relazioni di genere nel lungo periodo di incertezza, di caduta dei modelli ma anche di apertura di nuovi spazi di sperimentazione, cominciato con il superamento delle urgenze post-belliche, con le prime avvisaglie di un nuovo benessere e con i sommovimenti sociali e culturali che lo avevano accompagnato. La narrazione romanzesca, per lei, era stata fin ad allora, e sarebbe stata ancora, un modo per continuare a porsi queste domande senza sentirsi tenuta a dare delle risposte.

1. Ginzburg Natalia, "Donne e uomini", in Ead., Non possiamo saperlo. Saggi 19731990, a cura di Domenico Scarpa, Torino, Einaudi, 2001, p. 92. Le due citazioni precedenti si trovano rispettivamente a p. 90 e a p. 91. 
Nella lettura che ne ha dato Cesare Garboli, Natalia Ginzburg è la narratrice dell'“impatto della Ragazza col mondo, lo strappo che fa nascere la vita dal sangue e, di colpo, la fa essere adulta" nell'"urto che il corpo ancora inarticolato nella tana riceve dal contatto col mondo (l'Altro, il Maschio)"'. Da questa premessa discende una lettura complessiva condotta sulla falsariga di una sorta di binarismo essenzialistico rovesciato di segno (il "secondo sesso" non è più la donna, ma l'uomo) e tuttavia imperniato sulle stesse coppie oppositive biologico-antropologiche: le viscere e la mente, la natura e la storia, la tribù e la società ${ }^{3}$.

Mentre il polo femminile che regge questo schema è costretto nella fissità, ma anche nella concretezza, di uno stereotipo e del suo opposto (le "ragazze che sanno valutarsi, far valere il sesso e far brillare il corpo" e le "ragazze impacciate, chiuse, introverse [...] dalle camicette di piombo e dalle sottane di piombo"4), il polo maschile ha la duttilità, ma anche l'evanescenza, di un fantasma dell'immaginario (il Maschio), del quale i tipi maschili che compaiono effettivamente nella narrativa ginzburghiana non sono che il calco vuoto ("il requiem sulla virilità, la nostalgia del padre, l'eclisse del maschio"5) o il rovescio degradato: "maschi-ombre, visi pallidi e esangui, uomini in vago odore di omosessualità", che "si aggirano ai bordi dell'accampamento, ronzano intorno al fuoco, commerciano ricordi, stringono ambigue amicizie coi maschi patentati, poi, durante la stagione cattiva, riscompaiono inghiottiti dal deserto o dalla foresta da dove sono venuti"'.

Eppure in Un'assenza, il primo "racconto vero" di Natalia Ginzburg, che la stessa autrice data "luglio 1933", Maurizio, il protagonista-narratore, approfitta di un viaggio della moglie per dissezionare le ragioni non tanto di un matrimonio

2. Garboli Cesare, "Prefazione" a Ginzburg Natalia, Opere, vol. I, Milano, Mondadori, 1986, pp. XIX-XX.

3. Questa visione dicotomica funziona come chiave interpretativa anche di un romanzo tardo come Caro Michele: cfr. ID., "Introduzione" a GINZBURG Natalia, Caro Michele [1973], Milano, Mondadori, 1977, pp. V-XVII. Per non parlare della scrittura saggistica ginzburghiana, la cui varietà e ampiezza cronologica sono ricondotte a un immutabile "punto di vista femminile, viscerale, primitivo, diverso, uterino, sconosciuto all'uomo" (ID., "Prefazione" a GINZBurg Natalia, Opere, cit., p. XXV). Sarebbe interessante, anche se non è questa la sede per farlo, leggere Garboli alla luce delle categorie di genere con le quali lui stesso ha letto i "suoi" autori e soprattutto le "sue" autrici.

4. Ibid., p. XXI.

5. Ibid., p. XXVIII.

6. Ibid., p. XXX.

7. Ginzburg Natalia, "Prefazione" a Ead., Cinque romanzi brevi [1964], Torino, Einaudi, 1993, p. 5. 
senza amore quanto di un'identità maschile intimamente vacillante, che solo l'adeguamento meccanico agli usi della maschilità egemone (in questo caso il tradimento "a freddo" con una giovane donna, consumato come rivalsa per il sospetto adulterio della moglie) è in grado di riconfermare nelle proprie certezze; e già nei romanzi brevi degli anni Quaranta e Cinquanta, da La strada che va in città a Sagittario, i ruoli e i modelli di genere si sovrappongono, si mescolano e si confondono, in un continuo variare del sistema dei personaggi che sembra retto, più che dalla rigidità delle opposizioni binarie, da una sorta di logica combinatoria - la più coerente, del resto, con un'idea della forma romanzo non solo come mimesi ma anche come sperimentazione di atteggiamenti psicologici e di relazioni interpersonali ${ }^{8}$.

Nell'immagine di se stesso a quindici anni rievocata da Maurizio in Un'assenza, "un ragazzo magro, con un largo grembiale turchino", che "non desiderava le donne", James Michael Fortney ha individuato una delle spie linguistiche dell'omosessualità del protagonista ${ }^{9}$. Probabilmente non è un caso che nell'ampiezza e nella libertà di questa ricognizione, la figura dell'omosessuale maschio compaia fin dalle prime prove e svolga una funzione progressivamente più cruciale, soprattutto a partire dagli anni Settanta ma con una anticipazione significativa all'inizio degli anni Cinquanta. Nelle pagine che seguono, intendo analizzarne la presenza e lo sviluppo in tre romanzi che la mettono al centro della narrazione - Valentino (pubblicato in volume nel 1957, ma comparso su Botteghe Oscure nel 1951), Caro Michele (1973) e La città e la casa (1984) - non solo nella sua funzione destrutturante dei modelli egemonici di maschilità, ma anche e soprattutto in quella strutturante all'interno della narrazione.

La prima difficoltà di questa indagine consiste nel fatto che in Natalia Ginzburg la ricorrenza del personaggio omosessuale va di pari passo con la sua scarsa connotazione, sia in senso "militante" sia in senso "simbolico". Nella letteratura italiana, che "almeno fino agli Sessanta, raramente ha osato rappresentare amori omosessuali" senza dover "fare i conti con enormi e spesso

8. Una ricognizione sistematica, anche se non sempre condivisibile, dei personaggi maschili nella prima Ginzburg è offerta da Bullock Alan, "Uomini o topi? Vincitori e vinti nei Cinque romanzi brevi di Natalia Ginzburg”, Italica, vol. 60, n. 1, Spring 1983, pp. 38-54. Sullo stesso argomento cfr. anche WeINSTEIN Jen, "Il maschio assente nell'opera narrativa e teatrale di Natalia Ginzburg", in Testaferri Ada (a cura di), Donna. Women in Italian Culture, Ottawa, Dovehouse Editions, 1989, pp. 89-98.

9. Cfr. Fortney James Michael, "Con quel tipo li’: Homosexual Characters in Natalia Ginzburg's Narrative Families", Italica, vol. 86, n. 4, Winter 2009, pp. 659660. Anche Casa al mare, del 1937, è oggetto di un'interpretazione analoga (cfr. ibid., pp. 660-661). 
insormontabili problemi di censura e di autocensura", sono state soprattutto le scrittrici ad aprire la strada alla rappresentazione di uomini omosessuali più "interessanti e complessi"10, lontani dagli stereotipi della retorica machista ma anche dalle tentazioni della vittimizzazione. La differenza di genere scioglie dai lacci dell'autobiografismo e permette a Ginzburg di situarsi al di qua delle ideologie, della morale, degli psicologismi e dei sociologismi, su un piano che è da un lato fenomenologico dall'altro squisitamente narratologico ${ }^{11}$.

All'inizio degli anni Cinquanta, in un momento in cui l'omosessualità maschile cominciava a entrare nel discorso pubblico nelle forme opposte dell'autoaffermazione stigmatizzazione omofoba ${ }^{12}$, Ginzburg sfaccetta il proprio personaggio attraverso una duplice strategia rappresentativa, che rispetto alla visione frontale privilegia la litote e la mediazione dello sguardo altrui. Le prime immagini che il testo offre di Valentino lo mostrano intento a guardarsi e a farsi guardare: "passeggiava davanti allo specchio prima con una sciarpa buttata intorno al collo e poi senza; e s'affacciava al balcone per farsi vedere dai bambini della portinaia"13.

Sono così fissati da subito i tratti più rilevanti del protagonista - l'infantilismo e il narcisismo - ma insieme ad essi è allusa anche la sua funzione narrativa. Valentino sembra esistere solo nel riflesso dello specchio perché è uno specchio. È proprio la sua scarsa autonomia di personaggio, la sua indeterminatezza adolescenziale, a renderlo disponibile agli investimenti emotivi e ai rispecchiamenti

10. Gnerre Francesco, L'eroe negato. Omosessualità e letteratura nel Novecento italiano, Milano, Baldini \& Castoldi, 2000, pp.19-20. Quest'ultima constatazione fa risaltare ancora di più i limiti del criterio di selezione degli autori, tutti uomini e quasi tutti omosessuali, presi in esame da Gnerre.

11. Non mi sembra sia stato sottolineato abbastanza, meno che mai valorizzato, il privilegio assoluto accordato da Natalia Ginzburg, nei suoi rari interventi di autocommento, alle implicazioni di natura "tecnica" del fatto letterario: alla genericità con la quale individua i propri temi - la vita collettiva, i rapporti umani, che danno tra l'altro il titolo a due suoi saggi - fa da contraltare un'attenzione costante e una consapevolezza acutissima degli aspetti narratologici: la posizione e lo statuto del narratore, la densità e la coerenza dell'intreccio, il ritmo e la frequenza dei dialoghi, il rapporto tra autobiografia e invenzione. La già citata "Prefazione" a Cinque romanzi i brevi ne offre una campionatura eloquente. Ma si veda anche "Il mio mestiere", in Le piccole virtù, Torino, Einaudi, 1962, pp. 73-90.

12. Cfr. Petrosino Dario, "Crisi della virilità e 'questione omosessuale' nell'Italia degli anni Cinquanta e Sessanta", in Bellassai Sandro, Malatesta Maria, Genere e mascolinità. Uno sguardo storico, Roma, Bulzoni, 2000, pp. 317-343.

13. Ginzburg Natalia, Valentino [1951], in Ead., Cinque romanzi brevi, cit., p. 160. 
identitari altrui ${ }^{14}$. Nelle fantasie del padre, Valentino è destinato a diventare un medico famoso, ma non ha alcuna vocazione né il minimo interesse; sfida la famiglia per sposare una donna più vecchia di lui, tanto brutta quanto ricca e tanto volitiva nella gestione dei propri affari quanto lui è pigro e inconcludente, ma non conosce la profondità dei sentimenti; tradisce la moglie con il cugino di lei, Kit, che dopo un breve fidanzamento con la sorella di Valentino, Caterina, si toglie la vita, ma è intangibile dalle passioni e dai rimorsi. Le sue uniche azioni consistono nell'impersonare ciò che di volta in volta gli viene chiesto di essere, sia il marito-figlio a cui Maddalena accarezza i capelli dopo una dura giornata di lavoro sia l'uomo di mondo, indifferentemente a proprio agio su una barca a vela, in sella a un cavallo o in giro per le capitali europee, in cui Kit immagina di trasformarlo. Nell'economia narrativa del romanzo, così come nel ménage delle sue due famiglie, quella d'origine e quella cui dà vita, occupa uno spazio vuoto, è un vuoto; un punto cieco che ha però il potere di catalizzare le proiezioni, le aspirazioni, i desideri, persino le identità sessuali degli altri, per poi metterne a nudo il conformismo, il velleitarismo e la fragilità ${ }^{15}$.

Se è vero che il suicidio di Kit, con la conseguente scoperta della sua relazione con Valentino, rappresenta il punto di svolta della narrazione, il nucleo semantico del racconto è da cercare altrove, nella frase che compare per ben due volte nell'incipit del racconto e che ricorre come un leitmotiv perdendo progressivamente di significato: "mio padre credeva che sarebbe diventato un grand'uomo". Eppure è proprio il padre, che da maestro di scuola aveva goduto di una qualche autorevolezza ma che in casa non riesce a portare a termine una frase senza inciampare nella balbuzie, a riconoscerne l'insensatezza nella lettera che scrive al figlio prima di morire: "si scusava d'aver sempre sperato che Valentino diventasse un grand'uomo; invece non c'era nessun bisogno che diventasse

14. Francesca Sanvitale lo definisce giustamente "un narcisista" che incarna "i sogni taciuti da tutti di una diversa vita fatta di bellezza, successo, eleganza, genialità (SAnvitale Francesca, "I temi della narrativa di Natalia Ginzburg: uno specchio della società italiana", in Natalia Ginzburg. La narratrice e i suoi testi, Roma, La Nuova Italia Scientifica, 1986, p. 26). Ma altrettanto legittimamente Maria Antonietta Grignani vede in lui una "vittima a modo suo di conformismi cui la sua 'diversità' non può rispondere" (Grignani Maria Antonietta, "Un concerto di voci”, in ibid., p. 45).

15. In un'intervista a Walter Mauro, Natalia Ginzburg sottolinea, purtroppo senza approfondirlo, proprio questo aspetto: "Valentino è un personaggio in qualche modo misterioso che non riescono mai a conoscere. [...] non è un interlocutore perché è talmente innamorato di sé che non può ascoltare il prossimo. È il centro del racconto: quello su cui si accentrano le speranze, gli interrogativi, e le delusioni di tutti" (MAuro Walter, "Walter Mauro parla con Natalia Ginzburg", in ibid., p. 62). 
un grand'uomo, e bastava che diventasse un uomo, né grande né piccolo: perché adesso era soltanto un bambino"16.

Per quanto ridimensionato, il mandato paterno rimane non meno problematico. Perché in un momento storico e in un contesto sociale in cui le relazioni di potere tra generi e generazioni esercitano una pressione normativa più apparente che sostanziale, i padri sono in grado ancora meno dei figli di dire cosa significhi "essere un uomo". Di questo vicolo cieco, l'unico a essere davvero consapevole è Kit, con la sua coscienza infelice e il suo disprezzo di sé, che tuttavia non gli impediscono di rifiutarsi a un matrimonio di facciata. A questa altezza cronologica, l'omosessuale maschio può "essere un uomo" soltanto nell'ipocrisia o nell'autocancellazione. Ecco perché il suicidio di Kit, più che la pointe tragica del romanzo, è solo una tappa che prelude al ritorno alla situazione di partenza: Valentino, cacciato di casa da Maddalena, torna a vivere con la sorella, "a ciondolare per casa nella sua vestaglia lacera, a fumare e a fare le parole crociate, lui che mio padre credeva che diventasse un grand'uomo", ma anche a portare fuori dalle mura domestiche il "suo passo così felice, trionfante e libero" 17 .

Fino a quel momento i racconti e i romanzi di Natalia Ginzburg avevano seguito un andamento lineare, più o meno precipitoso, verso un'acme drammatica, spesso coincidente con una morte violenta o prematura. Valentino si mantiene fedele solo in apparenza a questo impianto, del quale l'autrice comincia forse a percepire l'artificiosità, proprio mentre lo smentisce: l'impatto col mondo raramente è uno scontro frontale, più spesso è una negoziazione tra le inclinazioni personali e la norma, tra i desideri profondi e lo spazio sociale disponibile per realizzarli. La simmetria del sistema dei personaggi (Maddalena-Valentino, Caterina-Kit) e l'inafferrabilità di quello principale imprimono alla narrazione un falso movimento che è in realtà un girare a vuoto, una traiettoria a spirale intorno a un nucleo inerte ma vorace, che rallenta il tempo narrativo fino a bloccarlo - e la continuità nel tempo, la capacità di preservarla e di sapersi muovere in essa, sono appunto prerogative dei padri ${ }^{18}$.

Nonostante sia l'oggetto quasi esclusivo delle conversazioni altrui, Valentino non accede mai, nel corso di tutto il racconto, al discorso in prima persona. Non parla, è solo parlato. Il suo silenzio è, paradossalmente, la garanzia della

16. GinZBurg Natalia, Valentino, cit., p. 171.

17. Ibid., p. 193.

18. Cfr. Zoja Luigi, "L'eclissi dei padri", intervista a cura di Daniele Balicco, Allegoria, n. 61, pp. 141-151. Zoja riprende e sintetizza qui i punti chiave del suo Il gesto di Ettore. Preistoria, storia, attualità e scomparsa del padre, Torino, Bollati Boringhieri, 2000. 
sua libertà di essere quello che vuole e la reticenza, le perifrasi, gli eufemismi e le ellissi con le quali gli altri personaggi si affannano per dire il suo segreto senza dirlo non fanno che riconfermare la sua funzione, più che di personaggio autonomo, di reagente delle identità altrui ${ }^{19}$. Vent'anni dopo, nella pluralità di voci che risuonano in Caro Michele, la parola impronunciabile è venuta alla luce, declinata nella sua varietà sinonimica: "pederasta represso", "omosessuale", "frocio" "ambidestro" ricorrono nelle lettere e nei dialoghi, riferiti sia al protagonista sia al suo amico Osvaldo, con una naturalezza che non ha nulla di morboso o di deprecatorio; non sono uno stigma né un giudizio, semmai la spia linguistica di una difficoltà, che è soprattutto una difficoltà dei personaggi femminili, a stabilire con gli uomini una relazione dalla quale è esclusa la componente sessuale.

Se Caro Michele mettesse in scena soltanto "la dissolvenza dell'uomo come asse portante della famiglia" 20 non costituirebbe una novità rispetto alle opere precedenti. Invece il romanzo semi-epistolare del ' 73 segna un punto di svolta nel percorso creativo di Natalia Ginzburg, sul piano sia della struttura narrativa sia della riflessione sulla maschilità condotta attraverso il personaggio dell'omosessuale. Di Valentino, Caro Michele riprende la struttura circolare e l'andamento centripeto intorno a un nucleo vacante, esplicitandone però i significati con un'evidenza che ha del programmatico e che prelude alla natura eminentemente saggistica della Città e la casa.

La circolarità è sigillata da due morti: quella del padre di Michele all'inizio del romanzo, quella del figlio alla fine. Il destino tragico non è però il solo elemento, forse neppure il più importante, che sostiene la simmetria tra i due personaggi. Dopo la fuga di Michele da Roma, sua sorella Angelica ricorda l'ultimo incontro col padre appena morto: di quest'uomo che le è estraneo e che non sembra nutrire per lei il minimo interesse, rimangono nella sua memoria la "voce amara e gracchiante [...] lacerante e solitaria come un disco rotto" ${ }^{21}$ con la quale si compiace dei suoi brutti quadri. E da una lettera di Adriana a Michele si scopre che era stato sempre lui a imporre un irragionevole apartheid dei sessi al momento della separazione dalla moglie: alla madre le figlie, al padre il figlio, privilegiato anche post mortem nel testamento che gli assegna la parte più consistente dell'eredità. Se quest'uomo senza nome, identificato soltanto dai suoi

19. Per un'analisi accurata di questa strategia retorica si veda ancora FORTNEY James Michael, "Con quel tipo li': Homosexual Characters in Natalia Ginzburg's Narrative Families", cit., pp. 654-657.

20. Ponti Paola, “Non si amano soltanto le memorie felici'. Caro Michele di Natalia Ginzburg”, Italianistica, a. XXXIX, n. 3, settembre-dicembre 2010, p. 114.

21. Ginzburg Natalia, Caro Michele, cit., p. 40. 
ruoli familiari, ruoli che per altro si è dimostrato ampiamente incapace di assolvere, è una parodia della maschilità tradizionale, suo figlio è la parodia della parodia: nelle sue peripezie di randagio senza scopo, anche Michele dipinge brutti quadri, probabilmente ancora più brutti di quelli del padre, sposa una donna che lascia dopo otto giorni, costeggia i gruppi terroristici senza alcuna convinzione politica. Trasforma in una recita bambinesca le tradizionali dimostrazioni pubbliche della maschilità nei suoi ambiti privilegiati - la famiglia, il lavoro, la guerra - e così facendo ne neutralizza il potenziale simbolico.

Come Valentino, inoltre, anche Michele scava nella narrazione un centro vuoto verso il quale si protendono vanamente i suoi interlocutori; e come Valentino, "prende consistenza innanzitutto grazie alle lettere che gli vengono spedite" 22 , ossia in quanto oggetto della parola altrui. Ma la figura chiave del romanzo non è lui. Nel riproporre la duplice caratterizzazione del personaggio omosessuale, sdoppiato nell'eterno adolescente e nell'adulto dolorosamente consapevole, questa volta Natalia Ginzburg lascia che sia il secondo a occupare la scena e a imprimere il suo segno alla vicenda.

All'inizio del romanzo Osvaldo Ventura è una presenza defilata e ambigua all'ombra di Michele, tra il gregario e l'amante fiaccamente corrisposto; via via che il destinatario delle lettere si sfoca in una lontananza che non è solo geografica, Osvaldo diventa il mediatore tra due mondi, quello maschile e quello femminile, che la rottura tra i genitori di Michele ha allontanato come due pianeti fuori orbita, e "compensa la smaterializzazione degli uomini di famiglia garantendo una presenza quotidiana" ${ }^{23}$. Onnipresente nelle lettere e nella vita quotidiana di Adriana, infaticabile nel procurare a Mara, la ragazza-madre sbandata che rappresenta il corrispettivo femminile di Michele, piccoli lavori, alloggi e denari, legato all'ex moglie Ada da una complicità disincantata ma affettuosa e dedito alla cura della figlioletta, non solo Osvaldo non è più il soggetto maschile negato dalla norma, stretto nell'alternativa tra distruggere o distruggersi, ma è l'unica figura "paterna", per quanto irresoluta e opaca, sopravvissuta in un mondo senza uomini - un surrogato, probabilmente, ma come lui stesso scrive a Michele, "d'altronde noi tutti viviamo di surrogati"24.

Nel registrare i cambiamenti del costume a vent'anni di distanza da Valentino, Ginzburg tenta una strategia rappresentativa originale, per quanto non priva di

22. Ponti Paola, “"Non si amano soltanto le memorie felici'. Caro Michele di Natalia Ginzburg”, cit., p. 116.

23. Ibid., p. 120.

24. Ginzburg Natalia, Caro Michele, cit., p. 113. 
contraddizioni: da un lato rifugge lo stereotipo dell'omosessuale come maschio svirilizzato ma anche quello opposto, esibizionistico e ipersessualizzato; e nel tratteggiare il personaggio a partire non tanto dal suo amore per gli uomini quanto dalle sue relazioni con le donne, ne offre un ritratto inedito proprio perché rifiuta di far coincidere la sua identità con l'orientamento sessuale. Dall'altro lato, però, ne occulta e ne depotenzia un aspetto fondamentale: il suo personaggio omosessuale, a questa altezza cronologica, sembra prefigurare un nuovo modello di maschilità, ma è ancora un maschio senza corpo.

La portata e i limiti della svolta segnata da Carlo Michele emergono più chiaramente se lo si legge in parallelo con il suo romanzo gemello, La città e la casa, che rispetto al primo si presenta come un'operazione di sofisticato, deliberato manierismo e di estremismo radicale. Col manierismo, Natalia Ginzburg riscrive Caro Michele replicandone l'intreccio, ma aggiungendo a ogni evento la sua glossa ${ }^{25}$. Con l'estremismo, mentre sembra condurre allo stadio terminale sia la forma romanzo sia le relazioni di genere tra i personaggi, prefigura una possibile rifondazione di entrambi.

Nella sua configurazione "mista", a metà tra romanzo epistolare e narrazione in terza persona, Caro Michele introduceva nell'organicità della struttura romanzesca un elemento corrosivo che ne minava la capacità di dare un senso alla casualità e all'incoerenza delle traiettorie esistenziali dei personaggi. La città e la casa porta a termine questo processo con la definitiva scomparsa del narratore esterno: il caleidoscopio del romanzo si è rotto, lanciando i suoi minuscoli pezzi di vetro nelle direzioni più imprevedibili. Dall'esile traliccio narrativo di due dismissioni parallele - la vendita della casa di Roma da parte di Giuseppe prima di raggiungere il fratello a Princeton e quella delle Margherite, il casale nella campagna umbra dove Lucrezia e Piero hanno cresciuto i loro cinque bambini e accolto ogni fine settimana la vasta tribù degli amici fino al fallimento del loro matrimonio - si diramano traiettorie esistenziali accomunate dallo smarrimento e dalla ricerca di una protezione paterna ormai impossibile. Solo Alberico, il figlio omosessuale di Giuseppe, sembra avere l'energia per ricominciare: rico-

25. "L'evento e la sua glossa" distingue, secondo Garboli, la morte di Alberico in $L a$ città e la casa da quella di Michele nel romanzo del "73 (GArboli Cesare, "Prefazione" a Ginzburg Natalia, Opere, cit., p. XLIII). La consapevolezza programmatica con la quale la scrittrice amministra questo discorso di secondo grado, e che produce nel critico un sorprendente imbarazzo ermeneutico ("Io non sono tra quelli che amano l'ultimo libro della Ginzburg, ma non sono nemmeno tra quelli che non lo apprezzano [...] Io fatico a decifrarlo", p. XLIV), è a mio avviso una delle ragioni principali dell'importanza e della novità di un libro in cui scrittura narrativa e scrittura saggistica convergono esemplarmente verso il romanzo-saggio. 
struisce un nucleo familiare al di fuori dei legami di sangue e del tradizionale assetto borghese, dà il suo nome alla figlia di un'amica, che cresce come se fosse sua, scrive e gira un film di successo, è in procinto di ricomprare la casa del padre quando viene ucciso in una rissa tra spacciatori.

La sua posizione di rilievo e il suo ruolo di personaggio-cardine nella coralità del romanzo, addirittura più accentuata che in Caro Michele, trova conferma nella prima lettera che Giuseppe scrive a Lucrezia. È curioso, ma non senza significato, che al momento di congedarsi per sempre dalla sua ex amante, Giuseppe si dilunghi nel racconto della vita di Alberico, che per altro Lucrezia non ha mai conosciuto, in una sorta di compatto micro-romanzo biografico incastrato nella compagine frantumata del romanzo epistolare - come se spettasse solo ad Alberico il privilegio di avere una storia. E non è un caso che sia il padre a raccontarla. Il giovane omosessuale che vive apertamente il proprio amore per gli uomini non è più, come Valentino, lo specchio in cui il padre contempla il proprio vuoto; e neppure, come Michele, la caricatura inconsapevole di una maschilità fallimentare. Nella sua relazione indissolubile con la figura paterna, e più in generale con la paternità come unità di misura e criterio di verifica di ogni possibile modello di maschilità, Alberico inverte il vettore del rapporto: è lui, con il suo slancio vitale, la sua creatività e la sua capacità di accudimento, a lasciare a Giuseppe l'eredità di quella nuova idea dell'uomo sulla cui immagine Natalia Ginzburg aveva chiuso Donne e uomini.

E tuttavia, come Michele, anche Alberico muore in un modo tanto inaspettato quanto casuale. L'evento è lo stesso, ma qual è la glossa? È l'ammissione da parte dell'autrice del proprio atteggiamento ambivalente nei confronti della maschilità e delle sue rappresentazioni: da un lato, la sistematica decostruzione alla quale Ginzburg sottopone il modello patriarcale di maschilità non è separabile dalla sua difficoltà a credere in un'alternativa; dall'altro, l'ammissione della propria incapacità di dare un futuro alla nuova idea di uomo che lei stessa ha immaginato non le impedisce di liquidare senza alcun rimpianto quella vecchia.

Beatrice MANETTI

Università di Torino 
